\title{
RETRACTED ARTICLE: Imperialism in Bioethics: How Policies of Profit Negate Engagement of Developing World Bioethicists and Undermine Global Bioethics
}

\author{
Subrata Chattopadhyay • Catherine Myser • \\ Raymond De Vries
}

Received: 1 January 2013 / Accepted: 1 February 2015 /Published online: 22 July 2015

(C) Journal of Bioethical Inquiry Pty Ltd. 2015

It has been decided by the journal editors and Springer International Publishing AG to retract the above article. The retraction has followed a complaint that identified errors in statements and data presented in the article that were essential to its main conclusions.

The errors, which appear to have been made honestly, occurred in the abstract, the body of the text, and Table 1 :

- It was claimed that nine leading bioethics journals (The American Journal of Bioethics; Bioethics; Developing World Bioethics; The Journal of Law, Medicine and Ethics; the Journal of Bioethical Inquiry;

Electronic supplementary material The online version of the original article can be located at doi:10.1007/s11673-015-9654-4.

S. Chattopadhyay $(\bowtie)$

College of Medicine and Jawaharlal Nehru Memorial Hospital, The West Bengal University of Health Sciences, P.O. Kalyani, Dist. Nadia PIN 741235 West Bengal, India e-mail: linkdrsc@yahoo.com

S. Chattopadhyay

e-mail: linkdrsc@gmail.com

C. Myser

467 Vermont Ave, Berkeley 94707 CA, USA

R. De Vries

Center for Bioethics and Social Sciences in Medicine,

University of Michigan, Ann Arbor, MI, USA

R. De Vries

CAPHRI School for Public Health and Primary Care,

Maastricht University, Maastricht, The Netherlands
Medicine, Health Care and Philosophy; Cambridge Quarterly of Healthcare Ethics; The Journal of Medicine and Philosophy; and the Journal of Medical Ethics) do not participate in the Health InterNetwork Access to Research Initiative (HINARI) of the World Health Organization, a program that provides free or very low-cost online access to major journals for researchers in developing countries. In fact, these nine journals do participate in HINARI.

- It was claimed that full-text articles of fourteen bioethics journals are not available through the free digital archive of PubMed Central. In fact, selected articles and/or author manuscripts in these journals are available, which includes the nine journals listed above, as well as The Hastings Center Report; the International Journal of Feminist Approaches to Bioethics; The Journal of Clinical Ethics; the Kennedy Institute of Ethics Journal; and Theoretical Medicine and Bioethics. Additionally, The Journal of Medicine and Philosophy participates as an "NIH Portfolio" journal within PubMed Central, and the Journal of Medical Ethics deposits articles published via its "BMJ Open Access" option.

- It was recommended that "international bioethics journals published by commercial publishing houses like Springer and Wiley-Blackwell should join WHO's HINARI and make their published contents accessible to bioethics workers in LMICs." In fact, two out of the three Springer journals and three out of the four Wiley/Wiley-Blackwell journals listed in the article do participate in HINARI, and Springer 
and Wiley are two of the initial founding publishers of HINARI.

As a result of these errors, key conclusions of the article relating to the scope of access to bioethics journals and the policies and practices of publishers, journals, and scholars are no longer sustainable, necessitating its full retraction. In accordance with usual practice, the text will remain available as electronic supplementary material marked "retracted article."

The journal and the publisher apologize to readers and other affected parties for any inconvenience or embarrassment caused by the publication of this article. 